\title{
Le Nouvel humanisme: une critique du progressisme éducatif
}

\author{
Dominique Ottavi
}

\begin{abstract}
Resumé:
Le Nouvel humanisme est un mouvement de critique littéraire déployé du début du XXe siècle à la deuxième Guerre mondiale, qui a de nombreuses implications pour l'éducation. Nous abordons deux de ses principales figures, Irving Babbitt (1865-1933), et Norman Foerster (1887-1972) pour exposer ces enjeux. Pour les Nouveaux humanistes, l'enseignement des grandes œuvres a une portée morale et politique et ne doit donc pas être réservé aux spécialistes de la littérature ou de la philosophie. Cette étude doit être largement proposée, notamment au niveau de l'enseignement secondaire, dans une école démocratique. Pour cette raison l'éducation progressive est la cible de la critique, en tant qu'aspect d'une civilisation qui s'est dévoyée à partir de la modernité. Les progrès scientifiques ont engendré une puissance qui n'est pas accompagnée de sagesse, tandis que la réaction romantique au bouleversement de la culture par l'exploration de la nature a engendré un sentimentalisme stérile. C'est dans cette perspective que les œuvres doivent être analysées et transmises. Norman Foerster a composé et appliqué un programme pour l'université d'lowa qui donne une idée concrète de l'enseignement des Nouveaux humanistes. Devrait-on s'en inspirer pour former les sujets contemporains, manquant de repères dans le désordre du XXle siècle?
\end{abstract}

Mots clés:

nouvel humanisme; Norman Foerster; Irving Babbitt 


\title{
The New Humanism: the criticism of educational progressivism
}

\begin{abstract}
The New Humanism is a movement of literary criticism deployed from the beginning of the twentieth century to the Second World War, which has many implications for education. We discuss two of his principal figures, Irving Babbitt (1865) 1933), and Norman Foerster (1887-1972) to expose these issues. For the New Humanists, the teaching of "great books" has a moral and political significance and should not be reserved for specialists in literature or philosophy. This study must be widely proposed, especially at the level of secondary education, in a democratic school. For this reason, the Progressive education is the target of criticism, as an aspect of a civilization wich has reversed thte sense of Progress. Scientific progress has created a power that is not accompanied by wisdom, while the romantic reaction to the upheaval of culture through the exploration of nature has engendered sterile sentimentalism. It is in this perspective that works must be analyzed and transmitted. Norman Foerster has written and applied a program for the University of lowa that gives a concrete idea of the teaching of the New Humanists. Should we be inspired by them to give a "bildung" to the contemporary subjects, lacking landmarks in the disorder of the twentyfirst century?
\end{abstract}

Keywords: new humanism; Norman Foerster; Irving Babbitt.

\section{O novo humanismo: uma crítica do progressismo educativo}

Resumo: O novo humanismo é um movimento de crítica literária implantado desde o início do século XX, à segunda Guerra Mundial, que tem muitas implicações para a educação. Abordamos duas das suas principais figuras, Irving Babbitt (1865- 1933) e Norman Foerster (1887-1972) para expor estas questões. Para os novos humanistas, o ensino das grandes obras tem um significado moral e político e não deve ser reservado aos especialistas da literatura ou da filosofia. Este estudo deve ser amplamente oferecido, especialmente ao nível do ensino secundário, numa escola democrática. Por esta razão a educação progressista é o alvo da críticas, enquanto aspecto de uma civilização que se desvendou a partir da modernidade. Os avanços científicos levaram a um poder que não é acompanhado pela sabedoria, enquanto que a reacção romântica à modificação da cultura pela exploração, explorando a natureza criou um sentimentalismo estéril. É nesta perspectiva que as obras devem ser analisadas e transmitidas. Norman Foerster concebeu e implementou um programa para a Universidade de lowa, que dá uma ideia concreta do ensino dos Novos humanistas. Dever-nos-iamos inspirar neles para repensar os temas contemporâneos, que carecem de referências na desordem do século XXI?

Palavras-chave: novo humanismo; Norman Foerster; Irving Babbitt.

\section{El nuevo humanismo, una crítica del progresismo educativo}

Resumen: El Nuevo Humanismo es un movimiento de crítica literaria desplegado desde principios del siglo XX hasta la Segunda Guerra Mundial, que tiene muchas implicaciones para la educación. Discutimos dos de sus figuras principales, Irving Babbitt (1865) 1933, y Norman Foerster (1887-1972) para exponer estos problemas. Para los Nuevos Humanistos, la enseñanza de las grandes obras tiene un significado moral y político y, por lo tanto, no debe reservarse a los especialistas en literatura o filosofía. Este estudio debe ser ampliamente propuesto, especialmente a nivel de educación secundaria, en una escuela democrática. Por esta razón la educación progresiva es el objetivo de la crítica, como un aspecto de una civilización que ha desorientada desde la modernidad. Los avances científicos han creado un poder que no está acompañado por la sabiduría, mientras que la reacción romántica a la agitación de la cultura a través de la exploración de la naturaleza ha creado un sentimentalismo estéril. Es en esta perspectiva que las obras deben ser analizadas y transmitidas. Norman Foerster ha compuesto y aplicado un programa para la Universidad de lowa que da una idea concreta de la enseñanza del nuevo humanista. ¿Debería inspirarse para formar temas contemporáneos, careciendo de marcas en el caos del siglo XXI?

Palabras claves: nuevo humanismo; Norman Foerster; Irving Babbitt. 
En quoi le Nouvel humanisme, un mouvement de critique littéraire d'origine américaine qui s'est déployé du début du XXe siècle à la deuxième Guerre mondiale, intéresse-t-il l'éducation aujourd'hui? Opposé à l'éducation progressive, il a, plus largement, mis en cause le darwinisme, le pragmatisme. Plus largement encore, c'est une modernité en rupture avec une conception de la formation héritée de l'humanisme qui est la cible de sa critique. S'il est tentant de ne voir dans le Nouvel humanisme qu'une pensée conservatrice et une réaction d'arrière-garde contre l'innovation, on doit cependant prendre au sérieux l'inquiétude qui l'anime : l'impact des avancées scientifiques sur la pensée et sur la culture, la disqualification des héritages du passé, l'irruption d'un idéal éducatif centré sur l'adaptation au présent, plutôt que sur la formation au long terme de l'individu (au sens de la Bildung). De ce point de vue, l'enjeu du Nouvel humanisme a été de repenser les contenus de l'enseignement, en relation avec cette notion de formation, en interrogeant les legs de la culture occidentale, en interrogeant les priorités de l'institution universitaire. Quelles études l'université doit-elle promouvoir dans un régime démocratique? Pourquoi le défi d'éduquer un peuple de citoyens doit-il éviter de s'affadir dans une adaptation présentiste aux besoins supposés des individus ? Ces questions nous concernent autant que lorsque David Hoelever, pour justifier son ouvrage sur le Nouvel humanisme, louait la puissance de diagnostic de ces auteurs (Hoelever, 1977, p.VIII). Après avoir précisé qui sont les nouveaux humanistes, au moins à travers ses principales figures, nous verrons en quoi les enjeux théoriques des études littéraires s'articulent à une vision critique de l'éducation démocratique promue dans différentes figures du « progressivisme».

\section{Figures du Nouvel humanisme}

Irving Babbitt (1865-1933), fondateur du Nouvel humanisme, diplômé de Harvard en 1899, après avoir étudié à la Sorbonne en 1891, a commencé sa carrière en enseignant la littérature classique au collège de Montana. II a ensuite enseigné le français et la littérature française à Harvard de 1894 jusqu'à sa mort. II attachait une grande importance au niveau du collège, creuset de l'éducation démocratique, terreau d'une culture commune en amont de la spécialisation, qu'il estimait légitime au niveau doctoral. En 1908, son essai Literature and the American College, assura sa réputation de polémiste ; l'ouvrage relie la question des programmes et du rôle des études à ce niveau à son interprétation de l'évolution culturelle et des enjeux de la critique littéraire au XXe siècle.

II est révélateur que Paul Elmer More (1864-1937), disciple de Babbitt, diplômé également de Harvard et spécialiste des langues orientales, ait considéré son maître comme " un repère indiquant toujours la direction de Rome» (More cité par Hoelever, 1977, p.11). Insatisfait comme lui du système universitaire, il se tourna vers le 
journalisme, et devint rédacteur en chef de la revue The Nation. Ses articles furent réunis sous le titre: Shelburne Essays.

Parmi les élèves de Babbitt, Stuart Pratt Sherman (1881-1926), et Norman Foerster (1887-1972) sont représentatifs d'une seconde génération. Sherman fut directeur du Herald Tribune, quant à N.Foerster, il devint chef de file du Nouvel Humanisme dans les années 1930, après la disparition de son maître. II s'est illustré à l'Université d'lowa, où il a dirigé l'école des Lettres et des Sciences (the School of Letters and Science) pour la restructurer pendant un temps selon ses propres idéaux. L'expérience s'interrompit, signant la fin de l'influence du Nouvel humanisme sur les réformes universitaires, quand Harry K. Newburn, un adversaire adepte de l'éducation progressive de John Dewey, devint doyen du collège des arts libéraux. L'argument de l'adaptation aux besoins des étudiants revint sur le devant de la scène, au détriment du programme humaniste. Parce que les deux prétendaient défendre un enseignement supérieur démocratique, le combat des nouveaux humanistes nous invite à redéfinir nos priorités pour la transmission de la culture et notre conception même de la démocratie.

\section{Humanisme et «humanitarianism»}

Pour les Nouveaux humanistes, les enjeux de la littérature dépassent l'esthétique et la linguistique, car sa portée s'étend jusqu'à la morale et politique. Un argument souvent invoqué rappelle l'opposition entre la raison et l'imaginaire que Gaston Bachelard nous a rendue familière: la littérature représente la créativité. Elle résiste à l'hégémonie de la science qui recherche la preuve et l'objectivité au détriment de la sensibilité. Mais ce constat résulte, plus profondément, de l'orientation de l'histoire dans une société dominée par le pouvoir de la technique et le matérialisme, et où règnent les doctrines du darwinisme, du pragmatisme, du behaviorisme. Ces doctrines scientifiques, surtout quand elles prétendent expliquer l'esprit humain, conduisent à dénier la liberté et la spiritualité. La création est un pouvoir qui, pour les nouveaux humanistes, ne peut être ramené à la causalité par les sciences de la nature. Ils assument le dualisme en affirmant que l'humain ne se réduit pas à son être biologique. Or, pour le darwinisme, la pensée est un résultat du devenir évolutif ; pour le pragmatisme, l'adaptation est la principale motivation de l'activité ; quant à la psychologie expérimentale, elle récuse l'étude du psychisme par le recours à l'intériorité, au profit de l'observation des conduites, des réactions. Tous conspirent en faveur d'un réductionnisme qui ne laisse pas de place à l'imprévisible, à la gratuité, à la création, qu'il s'agisse d'œuvres ayant une valeur pour elles-mêmes, ou d'actes libres, qui passent outre les déterminismes et l'apparente utilité. A partir de là, I. Babbitt considère que le dualisme ne vise pas tant une rupture entre la matière et l'esprit qu'une différence irréductible entre le domaine de la nature et celui de la culture ou de la civilisation dont les créations qui ne se réduisent pas à la 
production, dans le domaine matériel, ni à la recherche de la preuve dans le domaine de la théorie (Hoelever, 1977, pp. 41-47).

Du point de vue de l'éducation, le naturalisme est incarné par John Dewey, mais ce dernier est considéré comme l'héritier de cette tendance plus vaste au réductionnisme de la culture occidentale. I. Babbitt identifie dans cette situation l'aboutissement d'un processus de longue durée, qui a engendré au passage deux formes opposées mais complices de perversion de la culture (Koons, 2010, pp. 198-207): I'humanisme «baconien» et l'humanisme «sentimental».

L'humanisme baconien est ainsi nommé et symbolisé d'après Francis Bacon, acteur de l'émergence des sciences modernes dès le XVle siècle. Mais ce dernier n'est pas la cause ou le seul responsable du mouvement qui a entraîné la pensée dans la direction de l'exploration et de la maîtrise de la nature. Les conquêtes de la science ont à partir de là engendré la croyance au progrès, alors que c'est plutôt au triomphe de la libido sciendi que l'on assiste d'après I.Babbitt: l'émergence d'une connaissance dépourvue de finalité, ou dont le but est cette poursuite elle-même, dans une fuite en avant, que la spécialisation croissante accélère.

L'hégémonie de la méthode scientifique, ainsi inaugurée, a engendré en retour une réaction dont Rousseau est le meilleur représentant (Babbit, [1919], 2009). Paradoxal penseur des Lumières, Rousseau est aussi le premier Romantique : avec lui surgit, en face de la froide raison, l'exaltation du sentiment, érigeant la liberté individuelle, le sentiment intérieur de la nature et l'attirance spontanée vers le Bien, contre cette emprise. A la fois par sa vie et par sa personnalité et par son œuvre, Rousseau est considéré par I. Babbitt comme un «impressionniste moral» (Babbit, [1919], 2009). Ces deux attitudes opposées et leurs figures emblématiques sont pourtant liées : ce sont des naturalismes, qui renvoient une partie des acquis de l'esprit humain, soit dans les limbes de la pensée non scientifique, soit du côté d'une pensée fautive et d'un progrès mensonger qui mènent l'humanité à sa perte.

Ces attitudes à la fois opposées et complices sont désignées toutes deux par l. Babbitt avec le terme dévalorisant humanitarianism : les deux pèchent par optimisme envers une nature humaine affranchie des normes et des repères et du patrimoine hérités de la culture classique.

Sous l'égide de Bacon, l'excès de confiance «humanitaire» concerne le pouvoir de la raison et la croyance au Progrès: la raison s'affranchit, alors, des autorités philosophiques et religieuses pour placer l'homme dans une position démiurgique. S'ouvre une ère où le progrès matériel et la domination de la nature produisent une émancipation dangereuse, car ils s'effectuent sans la sagesse qui permettrait d'en user (Babbitt, cité par Hoelever, 1977).

Du côté rousseauiste, se développe l'idée que la nature sauvage, l'enfant, incarnent la nature primitive et authentique de l'humanité, et que l'émotion est la voie vers la 
vérité. Là encore il y a émancipation et affranchissement du poids de l'histoire, des autorités; c'est contre la culture que Rousseau érige la protestation de la nature humaine. Si sincérité et vie intérieure deviennent la pierre de touche de l'authenticité qui persiste, comme la statue de Glaucus, sous les concrétions de la civilisation, c'est le sentiment qui peut être exalté sans limites. Cette attitude a eu de larges prolongements esthétiques dans le mouvement romantique, et en philosophie : alors que Bernardin de Saint-Pierre a prolongé Rousseau sur le mode lyrique, Auguste Comte, à la fois fasciné par le progrès scientifique et sentimentalement exalté, a poussé plus loin cette forme d'enthousiasme avec sa religion de l'Humanité. Ceci est qualifié par I.Babbitt d'enthousiasme humanitaire. (Babbitt, 1908, pp.4-7).

L'Humanitarianism et les " humanitaires " s'éloignent donc de l'humanisme: là se trouve l'origine de l'appellation de New Humanism, le néo-humanisme. En effet, par rapport à l'hybris contenue dans les idéaux «humanitaires» qu'en est-il de l'humanisme classique ? Celui qui remonte à des auteurs anciens tels que Cicéron, dont les œuvres ont été transmises et ont nourri la culture de la Renaissance, ne peuvent être qualifiées du même vocable que ces produits tardifs d'une crise de civilisation. En effet, l'humanisme recherche la maîtrise de soi contre la tyrannie de l'impulsion, ainsi que la sagesse pratique, les principes de l'action aussi bien que les normes de la civilité.

Dans cette analyse, la portée de l'opposition entre humanisme et "humanitaire» ou humanitarianism doit être examinée du point de vue moral, mais aussi du point de vue de la formation de l'individu. En effet, l'individu moderne se trouve, d'une part, confronté à une énorme quantité de savoir qui ne cesse de se ramifier en spécialités, en parts de connaissance qui ne sont plus articulées dans une vue d'ensemble bien qu'elles partagent l'ambition d'appliquer des méthodes scientifiques, et qu'elles proclament leur utilité. De ce point de vue, l'hyperspécialisation universitaire concrétise un problème de fond, l'atomisation des domaines, chacun prétendant, de plus en plus loin des autres, appliquer la méthode scientifique. Comment l'individu peut-il s'équiper du mode d'emploi qui lui permettrait de s'y orienter ? Par ailleurs, ce même individu est en quelque sorte abandonné à lui-même et à ses impulsions, à ses sentiments à fleur de peau, confronté à une responsabilité sans normes, sommé d'être son propre guide. Sur ce point, les analyses d'l.Babbitt ne peuvent-elles être rapprochées, malgré le risque d'anachronisme, de celle d'Ehrenberg lorsqu'il parle de la «fatigue d'être soì?

\section{Littérature, critique, morale}

Dans ces conditions, quel peut être le rôle de la littérature? Et celui de la critique littéraire? Cette analyse sur le long terme du développement de théories qui constituent des paradigmes scientifiques en même temps que des modèles psychologiques 
et politiques, lui accorde un double rôle. Tout d'abord, la littérature témoigne du mouvement de la pensée, ses créateurs formulent et rendent sensibles les mutations de la culture. Par là-même, la littérature acquiert un pouvoir de formation. Cependant, les œuvres ont besoin d'être transmises par une médiation, interprétées, pour prendre sens.

Pour illustrer le premier point, en tant qu'exemple, l'œuvre de Zola a une grande valeur: le «roman expérimental» porte la marque des sciences du vivant, et on sait à quel point les théories héréditaristes imprègne le cycle des Rougon-Macquart. Plus précisément, le style réaliste de Zola traduit par le procédé littéraire de la description la valeur accordée par les sciences à l'exactitude des faits, et le destin des personnages les montre soumis à la causalité (Hoelever, 1977, p. 111 sq). Autre volet de la modernité, toute la littérature romantique, de Bernardin de Saint-Pierre jusqu'à Keats, illustre le règne de l'émotion et l'intériorité magnifiée (Hoelever, 1977, p. 44 sq).

Ces exemples peuvent faire penser que l'approche de Babbitt pèche par réductionnisme, faisant des œuvres des «reflets» ou illustrations de leur époque. Mais c'est là que le rôle de la critique littéraire prend le relais: l'étude des œuvres doit les relier à un contexte, les situer dans I'histoire; cependant, ces éléments de compréhension ne sont pas suffisants. Cette approche scientifique révèle ses limites chez Taine par exemple, conduit à nier la singularité des œuvres (Hoelever, 1977, p. 69). Or, la critique littéraire doit aussi contribuer à mettre en valeur la singularité de l'œuvre, en montrant qu'elle est le produit non d'une causalité qui en épuiserait le sens, mais de l'intelligence créative des artistes. Leurs œuvres entraînent la pensée dans le sillage de la sensibilité, elles s'adressent au sensible mais accroissent en même temps l'intelligibilité du monde. S'il revient à la critique de révéler leur perfection formelle, et leur contexte historique, pardelà l'écart temporel qui peut les rendre difficiles d'accès, elle doit aussi révéler le sens qu'elles conservent par-delà les circonstances de leur apparition.

Dans cette mesure, les études littéraires, comme la critique, assument une médiation. Elles contribuent à conserver, à transmettre, et à identifier aussi les «grandes œuvres» (the Great Books). En effet tout n'est pas d'égale valeur, et le plus souvent c'est à juste titre que les lecteurs ont permis à certaines œuvres de franchir les siècles. Ce travail de la mémoire doit être entretenu mais aussi poursuivi, en prenant en compte les créations contemporaines, et éventuellement des oublis et injustices. Ce rôle attribué à la critique, et ensuite à l'enseignement, interdit de considérer les néo-humanistes comme de simples conservateurs. Si les œuvres du passé sont dignes d'être transmises, les classiques doivent être complétés par les œuvres du présent. Leur liste n'est jamais close, et elle peut toujours être révisée. La critique littéraire telle que l'envisage I. Babbitt et les autres néo-humanistes a une portée axiologique: toutes les grandes œuvres méritent attention, mais il faut analyser leur apport à la culture et les faire vivre. C'est une reconstruction de la culture que visent les néo-humanistes par ce travail, par-delà le double mouvement de la modernité et de ses illusions. 
Certains écrits sont en quelque sorte les symptômes d'un mal, expressions nécessaires, et parfaits en leur genre, de l'échec de la modernité. L'exaltation intempérante des sentiments peut donner des chefs-d'œuvre, ils abondent d'ailleurs dans la littérature et la poésie romantique. Les écrivains réalistes qui portent une attention quasi scientifique aux descriptions de l'expérience, comme Zola, mais aussi Jack London ou Thoreau, témoignent, chacun à leur façon, du désarroi des individus modernes, confrontés à l'univers matériel dans toute sa brutalité, expérimentant en quelque sorte les limites de l'idée de progrès et de la foi en l'humanité. Un autre type d'œuvre donne les moyens de dépasser ce mal-être et possède un pouvoir de reconstruction. C'est là que la critique littéraire et l'enseignement s'en emparent pour compléter la formation des individus. Platon ou Cicéron, mais aussi Goethe, tracent une voie qui permet de construire le moi, en se confrontant à des conflits de valeurs, à l'expérience de l'altérité, à travers la fiction et l'écriture. Ainsi, les œuvres ne « font " pas la morale, elles ne sont pas pourvoyeuses de leçons, elles permettent simplement de se confronter à des cadres de référence et à exercer le jugement, forgeant le sens de la responsabilité ; en cela, elles permettent la connaissance de soi. Ces œuvres font davantage qu'instruire, elles transforment.

C'est donc ce processus que doit viser l'étude. La critique et l'enseignement qui s'en nourrit auront alors une portée morale, loin d'une "science " de la littérature qui s'ajouterait à la liste des domaines de connaissance. Alors, la réflexion sur le sens des œuvres, sur le rôle de la critique, sur la nécessité de refonder la culture contemporaine en proie à l'autoritarisme de la science et à l'errance subjective, ouvre à une réflexion sur l'enseignement, l'université, et sur le défi d'une éducation démocratique. Cette théorie de la culture ne se développe pas dans la pure abstraction. Bien au contraire, l'intervention des néo-humanistes se situe dans un processus de rationalisation du système éducatif aux Etats-Unis, accompagné de débats souvent polémiques, qui opposent des conceptions différentes de finalités de l'éducation et des institutions éducatives, notamment de l'université.

\section{Les options, bonne ou mauvaise idée?}

II revient à I. Babbitt d'avoir déclenché les hostilités concernant la finalité des études pour la jeunesse, en faisant la critique d'une innovation qui nous paraît aujourd'hui bien banale, mais qui marque une importante inflexion. Charles William Eliot, président de l'université de Harvard, introduisit dans son université la possibilité de choisir des options. En amont, il dirigea les travaux du Comité des Dix, chargé en 1892 de refonder sur des principes homogènes l'enseignement des High schools, tiraillé entre des conceptions élitaires ou démocratiques des études, et entre des conceptions opposées des programmes, selon la place reconnue aux humanités classiques ou à des 
enseignements utilitaires, visant d'abord l'insertion professionnelle. II s'agissait donc non seulement d'une réflexion sur le contenu des programmes, mais encore, sur les principes de l'orientation. Les travaux de ce comité produisirent l'idée de curriculum individualisé et de choix des options, le fameux Elective System. Le principe en est de laisser les étudiants libres de choisir des options dans leur cursus, qui cesse donc d'obéir à un programme entièrement imposé (Montagutelli, 2002; Alix, 2017). La phrase suivante est souvent citée au titre des polémiques engendrées par cette réforme :

"A well instructed youth of eighteen can select for himself a better course of study than any college faculty, or any wise man who does not know him and his ancestors and his previous life, can possibly select for him...every youth of eighteen is an infinitely complex organization, the duplicate of which neither does nor ever will exist» .

Ainsi chaque individu est unique et ses besoins pour sa formation ne sont pas assimilables aux besoins d'un autre. Le jeune est, d'autre part, considéré comme autonome, il est censé savoir, mieux que ses aînés, professeurs compris, ce qui lui convient. II doit donc être autorisé à faire des choix parmi des propositions dont la liste ne peut être infinie mais qui demeure par principe extensible. En tout cas, ces propositions, pour C.W. Eliot, devaient être plus adaptées au monde moderne que les programmes traditionnels et permettre plus d'ouverture sur les sciences et techniques. Ainsi, le principe du choix devait permettre non seulement de donner place à la singularité individuelle, aux goûts et capacités de chacun, mais encore, de faciliter le parcours des études à tous les jeunes quelles que soient leurs origines sociales, ethniques, etc.

Malgré ses bonnes intentions, cette innovation apparut aux yeux de I. Babbitt comme un concentré des problèmes de la culture moderne. D'abord, elle donne un exemple typique de sentimentalisme humanitaire, qui identifie les besoins de l'étudiant avec son idiosyncrasie : ressentir une attirance pour un sujet d'étude suffit-il à affirmer qu'une aptitude existe, ou que le besoin de formation soit réel ? La foi accordée à la nature humaine, le développement de l'esprit indépendamment de toute culture, n'abandonne-t-elle pas la formation aux initiatives désordonnées d'un jeune abandonné à lui-même? I. Babbitt voit là aussi le mirage d'une extension indéfinie des droits individuels, sans prise en compte d'intérêts communs au sein desquels ces droits puissent prendre sens.

Enfin, le défenseur des humanités accuse la nouvelle orientation de scientisme : donner plus de place aux connaissances scientifiques parce qu'elles sont plus utiles, plus adaptées au monde contemporain, n'est-ce pas céder à la croyance aveugle dans le progrès, à l'illusion baconienne?

Sur ces deux points, la confiance mise en la nature individuelle comme l'illusion du progrès technique et matériel, Babbitt décèle à juste titre l'influence d'Herbert Spencer. Le philosophe évolutionniste a, dans ses Essais sur l'éducation (Spencer, 1861), préconisé les savoirs scientifiques au détriment des humanités classiques et 
des disciplines littéraires. II appelait aussi, à l'instar de Rousseau, prétendait-il, à une réforme de l'éducation pour faire place à l'épanouissement de l'enfant et à la manifestation spontanée de ses aptitudes. Pour cette raison, Spencer s'est illustré par son opposition aux punitions, qu'il estimait faire obstacle au développement naturel. G.W. Eliot a, effectivement, voulu appliquer ces idées, ce que montre en particulier son «Introduction de 1911 aux Essais de Spencer sur l'éducation». II y préconise à son tour l'enseignement scientifique, surtout des sciences de la nature, ainsi que les méthodes libérales d'éducation, afin de laisser s'épanouir les aptitudes individuelles (Eliot 1911). II y prend également acte du recul inévitable de l'influence des classiques. Cependant, la pédagogie de John Dewey, davantage habitée par le pragmatisme que par le libertarisme de Spencer, tombera aux yeux de I. Babbitt dans les mêmes travers : I'éducation progressive ne considère-t-elle pas l'apprentissage comme un prolongement du développement naturel, plutôt que comme une imprégnation par la culture ?

\section{Norman Foerster: un exemple de programme humaniste}

Dans les années 1930, l'ancien élève d'l.Babbitt, Norman Foerster, prend la relève (pour peu de temps). II illustre la seconde génération des Nouveaux humanistes en mettant en pratique les idéaux du mouvement. Directeur de l'école des Lettres et des sciences de l'Université d'lowa, il en fait une structure qui regroupe les départements d'anglais, d'allemand, de littérature classique et des langues romanes. II introduit alors un programme universitaire nouveau.

En 1946, l'ouvrage The Humanities and the Common Man, N. Foerster justifie cette expérience et définit les buts de l'université. Selon lui, l'université doit " être un instrument de la démocratie américaine pour mettre les humanités à portée de l'homme ordinaire » (Foerster, 1946, p.V). Comme I. Babbitt, il considère que l'enjeu n'est pas seulement de ménager l'accès au savoir pour le plus grand nombre, mais d'assurer la formation des étudiants, dans un sens plus large : "Les cours doivent aider l'étudiant à construire une conception de la vie et une échelle de valeurs ». (Foerster, 1946, p.VII).

Un défi nouveau pour l'éducation s'est cristallisé pendant la seconde Guerre mondiale : résister à la culture de masse, conserver l'esprit critique face à l'influence des mass media, et des idéologies totalitaires. L'étudiant a, dès lors, besoin d'une éducation libérale, qui l'initie à la complexité dirait-on aujourd'hui en reprenant le vocabulaire d'Edgar Morin qui s'y prête rétrospectivement. N. Foerster appelle, en effet, à une formation qui prépare à mettre en doute les idées simplifiées par la communication de masse, à mettre à distance les émotions collectives suscitées et exaltées par le nazisme et le fascisme, les mythes qui prétendent abolir l'histoire et créer une humanité parfaite. Contre ces forces, il estime qu'il ne serait pas suffisant de vouloir former une élite distincte de la masse de la population, en s'imaginant que des gouvernants 
éclairés pourraient guider des populations passives : il faut forger pour les citoyens de la démocratie une connaissance commune, à la fois émancipatrice et partagée, fondée sur l'étude de ce que l'humanité a produit de meilleur .

La propagande et la culture de masse ne sont pas seules visées par la critique de $\mathrm{N}$. Foerster. En effet, il considère que des études réduites à des connaissances utilitaires et à la formation professionnelle constituent également un danger pour la démocratie (Foerster, 1946, p.36). En ce sens, les «éducationnistes », et d'abord, pour N.Foerster, les adeptes de l'Education progressive et de J.Dewey, peuvent tomber dans le travers de définir des objectifs en termes de capacités, en considérant la connaissance uniquement comme un moyen. Si la formation se réduit, d'une manière ou d'une autre, à un but limité en vue d'une tâche ou d'une fonction, cela conduit d'abord à considérer l'être humain lui-même comme un moyen et à lui dénier la liberté. De plus, cette restriction des ambitions amène à séparer le travail et le loisir, plaçant l'individu libéré des contraintes du travail face à un temps vide. Or, le loisir est un ancien défi pour l'éducation, dont l'importance renaît dans le monde moderne. Les nouveaux humanistes s'inspirent volontiers d'Aristote sur ce point, même si ce dernier théorisait le loisir dans la démocratie antique, où la liberté se payait de l'existence d'esclaves. Le rôle de l'université d'Etat, et principalement de l'undergraduate education, devra être pour N. Foerster de ranimer l'ancienne tradition des Humanités, dans la perspective d'une formation largement partagée, et en évitant le piège de la spécialisation prématurée, qu'elle soit professionnelle ou scientifique. Citant Emerson, N. Foerster estime que ce projet est réaliste dans le monde contemporain: «the great books he finds always contemporary, or rather timeless, because they impress us with the conviction that one nature wrote and the same reads»( (Foerster, 1946, p.VIII).

Compte tenu de ces arguments, N. Foerster n'hésite pas à proposer aux étudiants de l'université d'lowa:

- Les lettres gréco-romaines, les œuvres en langues romanes traduites

- L'histoire de l'humanisme

- La théorie et la pratique de la critique littéraire

- L'études des auteurs anciens tels que Homère, Platon, Chaucer

- Et aussi la littérature contemporaine.

Une communauté de préoccupations unit ces œuvres que les disciplines universitaires tendent à trop séparer, l'enjeu de leur étude est pour N. Foerster de les relier dans une même culture.

Cet idéal n'est-il trop difficile à atteindre pour la majorité des étudiants ? N. Foerster fait face au problème par des mesures proprement pédagogiques, comme l'usage de traductions pour privilégier le sens de textes en langues anciennes, par des arguments d'ordre éthique: l'effort demandé enseigne le contrôle de soi ; en outre, la connaissance 
de l'excellence est inhérente à la démocratie : pour l'attendre d'une élite dirigeante, il faut la connaître et savoir l'identifier. Enfin, du point de vue de l'étudiant moyen, cette fameuse excellence n'a pas à être exigée de chacun de la même manière, car chacun est appelé à faire valoir ses qualités propres plutôt qu'à atteindre un standard de l'excellence: "chaque homme ordinaire a des capacités hors du commun » dit N. Foerster, faisant ici référence à Jefferson (Foerster, 1946, p.VII). II convient d'ajouter que le programme néo-humaniste de N. Foerster n'est pas figé, non seulement il s'ouvre aux œuvres contemporaines, mais encore il prévoit d'inclure l'enseignement scientifique, non sans anticiper sur des débats de la fin du XXème siècle. II considérait en effet que les sciences de la nature gagnent à intégrer l'élément de la créativité qui est au centre de la culture humaniste. Les choix de l'université d'lowa provoquent la réflexion, posent et reposent la difficile question: quelles sont les connaissances que les générations présentes doivent estimer pertinentes pour la formation des générations nouvelles?

Encore aujourd'hui, l'enseignement se heurte à cette difficulté : distinguer des chefsd'œuvre dont l'enseignement doit assurer la transmission entraîne parfois une conception patrimoniale de leur valeur qui nuit à l'enseignement. En effet, si des œuvres se distinguent par leur beauté, leur accomplissement, parce qu'elles nous lèguent aussi un témoignage du passé, cela ne suffit pas toujours à justifier leur étude pour de nouvelles générations. Les enseignants entendent souvent cette question: à quoi cela sertil? L'argument du plaisir qu'on en retire n'est pas toujours le meilleur. Le plaisir promis ne se décrète pas, et, en outre, cet argument confine la lecture à un rôle distractif.

Les nouveaux humanistes apportent leurs réponses: la valeur des «grandes œuvres» n'a pas à être considérée comme seulement esthétique ou patrimoniale, elle réside aussi dans leur pouvoir de formation. Même lorsque leur étude tend vers la rigueur scientifique, cela ne doit pas se faire au détriment de l'éducation morale et de la formation du citoyen que l'on est en droit d'en attendre, pour un grand nombre de jeunes, et pas seulement pour les futurs spécialistes des disciplines littéraires.

Stuart Pratt Sherman, un représentant de la seconde génération des néo-humanistes, résume ainsi l'idée: "Je conçois la littérature comme un partenaire de la politique, de la religion et de la morale» (Sherman, cité par D. Hoelever, 1947, p.86). N. Foerster renforce ce point de vue, considérant la formation en tant que connaissance de soi et réalisation individuelle (Foerster, 1947, p.V). On comprend, à partir de là, que le rôle de l'université soit essentiel dans les premières années, l'«undergraduate education»; produire des spécialistes reste important pour l'avancée de la recherche, mais éduquer la jeunesse est un but tout aussi important pour l'université, en amont de la spécialisation, et qui s'ajoute à elle en pour forger la culture commune, ou la culture de l'homme ordinaire.

Le fait d'être partagée n'empêche pas cette culture d'être exigeante, elle doit éviter le piège d'une culture "générale » éclectique. Un corpus fait d'œuvres rassemblées au 
hasard, ou parce qu'elle sembleraient accessibles, ne soutient pas la formation. Les œuvres importantes ne se plient pas à ce désir de facilité, et le rôle de l'université n'est pas non plus de fabriquer une sorte de liste consensuelle pour doter les étudiants d'un bagage minimal au gré des changements de mode. Abaisser le niveau pour rendre la culture accessible, ou ne considérer que les gloires du présent sous prétexte qu'elles sont plus faciles d'accès, reviendrait à considérer que la majorité des jeunes ne sont capables ni d'effort ni de perspicacité, un a priori bien méprisant de la part des générations en place.

N. Foerster, dans Humanities and the Common Man (Foerster, 1947), dans Humanities and the Common Man, définit en quelques points cette culture commune : I'appropriation de l'héritage de la culture; le développement, dans l'individu, de sa propre humanité ; la préparation à affronter la relativité des valeurs ; et enfin, l'accommodement avec les problèmes désespérants posés au XXème siècle. Maintenant que nous avons atteint le XXlème, déjà chargé lui aussi de problèmes désespérants, l'enjeu paraît toujours d'actualité. La critique de certaines formes du «progressivisme» et le plaidoyer pour une certaine forme de conservation de la culture du passé ne signifient pas nécessairement conservatisme.

\section{Références}

Alix S. A. (2017). L'éducation progressiste aux Etats-Unis. Grenoble : PUG.

Babbit I. ([1919] 2009). Rousseau and Romanticism. New Brunswick, London: Transaction Publishers.

Babbit I. (1924). Democracy and Leadership. New York: Houghton Mifflin Company.

Babbit I. (1908). Literature and American College. Boston: Houghton Mifflin Company.

Babbit I. (1929). « President Eliot and American Education », Forum, 1, 1-10.

Becquemont D., Ottavi D. (dir.) (2011). Penser Spencer. Presses Universitaires de Vincennes.

Ehrenberg A. (1998). La fatigue d'être soi. Paris: Odile Jacob.

Eliot G.W. (1911). Présentation d'Herbert Spencer, Essays on education. London \& Toronto: JM Dent \& sons, New York : EP Dutton \& Co.

Eliot G.W. (1898). Educational Reform: Essays and Adresses. New York: Century.

Foerster N. (1946). The Humanities and the Common Man. Chapel Hill: The University of North Carolina Press.

Harris M. (1970). Five Counterevolutionists in higher Education. Oregon State university.

Hoelever jr.J.D. (1977). The New Humanism, A critique of Modern America. Charlottesville : University Press of Virginia.

Koons R. C. (2010). "The War of the Three Humanisms: Irving Babbitt and the Recovery of Classical Learning » Modern Age, Summer, 3 (52), 198-207.

Montagutelli, M. (2000). Histoire de l'enseignement aux Etats-Unis. Paris: Belin. 
Morin E. (1977). La méthode, 1. Paris: le Seuil.

Ottavi D. (2013). «L'autre Rousseau », In Anne-Marie Drouin-Hans, Michel Fabre, Denis Kambouchner, Alain Vergnioux (dir.). L'Emile de Rousseau: regards d'aujourd'hui. (pp.131-145). Paris: Hermann.

Rousseau, J. -J ([1755] 1971). Discours sur l'origine et les fondements de l'inégalité parmi les hommes. Paris: Garnier-Flammarion.

Spencer H. (1861). Education: intellectual, moral and physical, London: G.Manwaring.

\section{Dominique Ottavi}

Professeur en sciences de l'éducation à l'université Paris Nanterre. Ses recherches portent sur la philosophie de l'éducation, sur l'histoire des idées

éducatives en particulier l'influence de l'évolutionnisme dans les sciences humaines et sociales, ainsi que sur l'analyse des problèmes contemporains

de l'éducation.

Email: ottavi.d@orange.fr

\section{Adresse}

Dominique Ottavi 57 rue Boissonade 75014 Paris

Data de submissão: Março 2018 Data de avaliação: Agosto 2018

Data de publicação: Abril 2019 